\title{
Continuidade da assistência ao paciente pós-tratamento do infarto agudo do miocárdio: Revisão integrativa ${ }^{1}$
}

\author{
Continuity of patient care after treatment of acute myocardial infarction: Integrative review \\ Continuidad de la atención al paciente después del tratamiento del infarto agudo de miocardio:
} Revisión integradora

Recebido: 05/04/2021 | Revisado: 12/04/2021 | Aceito: 13/04/2021 | Publicado: 25/04/2021

\author{
Karina Nunes Porto \\ ORCID: https://orcid.org/0000-0003-2778-0775 \\ Universidade Federal de Pelotas, Brasil \\ E-mail: karinaporto24@gmail.com \\ Josiele de Lima Neves \\ ORCID: https://orcid.org/0000-0002-8754-059X \\ Universidade Federal de Pelotas, Brasil \\ E-mail: josiele_neves@hotmail.com \\ Lílian Moura de Lima Spagnolo \\ ORCID: https://orcid.org/0000-0003-2070-6177 \\ Universidade Federal de Pelotas, Brasil \\ E-mail: lima.lilian@gmail.com \\ Jéssica Stragliotto Bazzan \\ ORCID: https://orcid.org/0000-0002-8457-134X \\ Universidade Federal de Pelotas, Brasil \\ E-mail: jessica_bazzan@hotmail.com
}

\begin{abstract}
Resumo
Objetivo: Analisar a produção científica sobre a continuidade da assistência ao paciente pós-tratamento do Infarto Agudo do Miocárdio e as potenciais intervenções da enfermagem. Metodologia: Trata-se de revisão integrativa de literatura. As buscas foram realizadas nas bases de dados National Library of Medicine, Sistema Latino-Americano e do Caribe de Informação em Ciências da Saúde, Biblioteca Virtual Scientific Electronic Library Online, Web of Science e Scopus, em setembro de 2020. Descritores utilizados: Continuidade da Assistência ao Paciente; Infarto do Miocárdio; Atenção primária à Saúde, sendo selecionado ao final 31 artigos. Utilizou-se Análise de conteúdo. Foram elencadas três categorias: Identificação de fatores de risco associados às complicações após tratamento de Infarto agudo do miocárdio; Estratégias para auxiliar na redução das taxas de readmissão hospitalares pós-tratamento de Infarto agudo do miocárdico; e Continuidade da assistência por meio de cuidados e gerenciamento da adesão medicamentosa. Resultados: Perante os 31 artigos selecionados, identificou-se a existência de preocupação com a identificação precoce dos fatores de risco; criação de estratégias para reduzir a readmissão hospitalar e traçar planos de cuidados de acordo com a literatura internacional, porém, no Brasil a temática é incipiente. Evidencia-se carência de estudos que enfatizem a participação da Enfermagem para efetivação das estratégias no acompanhamento ao paciente pós infarto. Conclusão: Há um amplo engajamento na elaboração de estratégias para reduzir a readmissão hospitalar, a aplicação dos modelos de predição de fatores de risco é satisfatória, auxiliando no planejamento de intervenções de enfermagem, e elevando a sobrevida.
\end{abstract}

Palavras-chave: Continuidade da assistência ao paciente; Infarto do miocárdio; Atenção primária à saúde; Enfermagem.

\begin{abstract}
Objective: To analyze the scientific production on the continuity of assistance to patients after treatment for acute myocardial infarction and the potential interventions of the disease. Methodology: this is an integrative literature review. While you search for forms made in the databases National Library of Medicine, Latin American and Caribbean Health Sciences Information System, Virtual Scientific Library Electronic Online, Web of Science and Scopus, in September 2020. Descriptors used: Continuity Patient Assistance; Myocardial infarction; Primary Health Care, with 31 articles selected in the last year. Content analysis was used. There are three categories listed: Identification of risk factors associated with complications in the treatment of acute myocardial infarction; Strategies
\end{abstract}

\footnotetext{
${ }^{1}$ Extraído do Trabalho de Conclusão de Curso, intitulado "Continuidade da Assistência ao Paciente pós-tratamento do Infarto Agudo do Miocárdio e as potenciais intervenções da enfermagem: Revisão Integrativa”, apresentada a Faculdade de Enfermagem da Universidade Federal de Pelotas (UFPel), no ano de 2020.
} 
to help reduce rates of hospital readmission after treatment for acute myocardial infarction; and Continuity of care through care and management of medication administration. Results: After the 31 items selected, there was a concern with the early identification of two precipice factors; Creation of strategies to reduce hospital readmission and outline care plans according to the international literature, mainly, not for Brazil with themes and incipients. Studies lacking evidence that emphasize the participation of nursing in the implementation of strategies that do not accompany the post-infarction patient. Conclusion: We added information to the development of strategies to reduce hospital readmission, to the application of two models for predicting risk and satisfaction factors, helping in the non-planning of illness interventions and increasing survival.

Keywords: Continuity of patient care; Myocardial infarction; Primary health care; Nursing.

\section{Resumen}

Objetivo: Analizar la producción científica sobre la continuidad de la asistencia a los pacientes después del tratamiento por infarto agudo de miocardio y lasposibles intervenciones de la enfermedad. Metodología: se trata de una revisión integradora de la literatura. Mientras busca formularios realizados em las bases de datos Biblioteca Nacional de Medicina, Sistema de Información de Ciencias de la Salud de América Latina y el Caribe, Biblioteca Científica Virtual Electrónica en Línea, Web of Science y Scopus, em septiembre de 2020. Descriptores utilizados: Continuidad Asistencia al Paciente; Infarto de miocardio; Atención Primaria de Salud, con 31 artículos seleccionados em el último año. Se utilizo análisis de contenido. Se enumeran três categorías: Identificación de factores de riesgo asociados com complicaciones em El tratamiento del infarto agudo de miocardio; Estrategias para ayudar a reducir las tasas de reingreso hospitalario después del tratamiento del infarto agudo de miocardio; y Continuidad de la atención mediante la atención y el manejo de la administración de medicamentos. Resultados: Después de los 31 ítems seleccionados, hubo preocupación por la identificación temprana de dos factores precipitantes; Creación de estrategias para reducir El reingreso hospitalario y delinear planes de atención de acuerdo com la literatura internacional, principalmente, no para Brasil con temas e incipientes. Estudios carentes de evidencia que enfaticen la participación de la enfermería em la implementación de estrategias que no acompañan al paciente postinfarto. Conclusión: agregamos información al desarrollo de estrategias para reducir el reingreso hospitalario, a la aplicación de dos modelos de predicción de factores de riesgo y satisfacción, ayudando em la no planificación de intervenciones de enfermedad y aumentando la superviven.

Palabras clave: Continuidad de la atención al paciente; Infarto de miocardio; Primeros auxilios; Enfermería.

\section{Introdução}

As síndromes coronarianas agudas (SCA) correspondem a grande maioria dos eventos cardiovasculares fatais. No Brasil, o IAM é responsável por 30\% das mortes, com maior ocorrência na faixa dos 60 anos, caracterizado por uma isquemia do miocárdio ocasionado pela redução ou interrupção total do fluxo sanguíneo coronário devido à aterosclerose coronariana com oclusão das artérias coronárias, assim acarreta déficits nas condições fisiológicas, sociais e laborais, com consequente baixa qualidade de vida (Troncoso et al., 2018).

De acordo com a Organização Mundial da Saúde (OMS) 75\% da mortalidade cardiovascular pode ser diminuída com mudanças no estilo de vida (OMS, 2017). Para isso, faz-se necessário a intervenção de uma equipe multidisciplinar de forma contínua, implementando estratégias de controle dos fatores de risco modificáveis como o tabagismo e obesidade, promovendo assim a prevenção primária (Simão et al.,2013).

No Brasil, a Atenção Primária à Saúde (APS) é a porta de entrada principal e o centro de comunicação da Rede de Atenção à Saúde. Esta rede objetiva melhorar o acesso e eficácia do Sistema Único de Saúde. Além de organizar o fluxo dos pacientes, a APS deve também ser responsável pelo usuário em todos os pontos da rede, produzindo assim um cuidado compartilhado e continuado. Neste sentido, a efetividade do sistema de referência e contrarreferência, articulação e comunicação entre os serviços, constitui o principal elemento para integração das redes de atenção à saúde (Schimith et al., 2014).

Para a efetividade dos cuidados, a APS deve apresentar atributos essenciais como a continuidade e longitudinalidade. Neste sentido, a manutenção do acompanhamento pós-tratamento do IAM é essencial para evitar complicações e um novo infarto. Diante deste contexto a enfermagem exerce papel fundamental, atuando na elaboração de estratégias para melhoria da 
qualidade de vida, mudança nos hábitos alimentares e adesão ao tratamento medicamentoso (Vargas et al., 2017). A enfermagem contribui tanto no desenvolvimento de ações assistenciais quanto educativas, atendendo o usuário em suas diferentes fases da vida, assim contribuindo para um cuidado continuado e longitudinal (Baratieri \& Marcon, 2011).

Desta forma, destaca-se a relevância de identificar as evidências disponíveis na literatura nacional e internacional sobre a continuidade da assistência ao paciente pós-tratamento de IAM, com vistas a destacar estratégias relevantes de cuidado, que favoreçam a preservação da saúde e acompanhamento permanente ao paciente, a fim de evitar agravamento da condição de saúde.

Com base no exposto, este estudo objetivou analisar a produção científica sobre a continuidade da assistência ao paciente pós-tratamento do Infarto Agudo do Miocárdio e as potenciais intervenções da enfermagem.

\section{Metodologia}

Trata-se de uma revisão integrativa (RI) com análise sistemática da literatura. A revisão integrativa foi desenvolvida em cinco etapas: definição da pergunta norteadora, assim delimitando o tópico de interesse da pesquisa; busca e seleção dos estudos primários, com o estabelecimento de critérios de inclusão e exclusão, o cruzamento na base de dados, a organização do banco de referências e seleção dos estudos para integrar a pesquisa; extração dos dados dos estudos primários; avaliação crítica dos estudos primários; a síntese de conhecimento; e por fim na última etapa apresenta-se os resultados (Mendes, Silveira \& Galvão, 2019).

Tem-se como pergunta norteadora: "Quais as evidências disponíveis na literatura científica sobre a Continuidade da Assistência ao Paciente pós-tratamento do Infarto Agudo do Miocárdio, e as potenciais intervenções da enfermagem?". Utilizou-se o acrônimo PICO (P- Paciente, Problema ou Grupo; I- Intervenção; C- Controle ou Comparação; e O- Outcomes, Desfecho ou Resultado). Para a presente pesquisa, os seguintes elementos foram avaliados: P- Paciente pós-tratamento para IAM; I- Continuidade da assistência; C- Potenciais intervenções da enfermagem; O- Impacto na redução de complicações.

Os descritores utilizados foram previamente consultados nos dicionários Medical Subject Headings (MeSH) e nos Descritores em Ciências da Saúde (DeCS). Com o uso do operador booleano "AND”, utilizou-se os seguintes descritores: Continuity of Patient Care; Myocardial Infarction; Primary Health Care.

Utilizou-se como critérios de inclusão: artigos originais publicados a partir do ano de 2015 até setembro de 2020, disponíveis na íntegra que abordassem a continuidade da assistência ao paciente pós-tratamento do infarto agudo do miocárdio, e as potenciais intervenções da enfermagem; publicados nos idiomas português, espanhol ou inglês. Os critérios de exclusão adotados: revisões de literatura, cartas ao editor e as duplicidades.

As bases de dados consultadas foram a National Library of Medicine (PubMed), o Sistema Latino-Americano e do Caribe de Informação em Ciências da Saúde (Lilacs), a Biblioteca Virtual Scientific Electronic Library Online (Scielo), a Web of Science e a Scopus. A coleta de dados foi realizada por dois revisores para garantir que todos os artigos que atendessem aos critérios de inclusão e exclusão, em caso de discordância foi solicitado avaliação de um terceiro. Utilizou-se como gerenciador de referências o software Mendeley.

Após a seleção dos estudos, para extração dos dados foi utilizado um instrumento construído e validado por Ursi (2006), contendo os seguintes itens: nome da pesquisa; autoria; revista de publicação; ano de publicação; objetivos do estudo; descrição metodológica; características da amostra; nível de evidência; resultados e conclusões.

Em relação ao nível de evidência das publicações, utilizou-se a proposta de Stillwell et al. (2010)que avalia a hierarquia dos estudos em níveis que podem variar de I a VII: Nível I - síntese de evidências de todos os randomizados relevantes, ensaios controlados; Nível II - Experimento em que os sujeitos são randomizados a um grupo de tratamento ou grupo de controle; Nível III - Experimento em que os assuntos não são aleatórios atribuídos a um grupo de tratamento ou grupo 
de controle; Nível IV - Evidências provenientes de estudos de coorte e de caso-controle bem delineados; Nível V - Síntese de evidências qualitativas ou descritivas estudos para responder a uma questão clínica; Nível VI - Evidências derivadas de um estudo descritivo ou qualitativo; Nível VII - Opinião oficial do comitê de especialistas.

Para a análise e interpretação dos artigos foi utilizada a proposta de Bardin (2013), composta de um conjunto de técnicas de análise do conteúdo das publicações, as quais permitem a inferência de conhecimentos para auxílio na avaliação dos resultados.

Respeitou-se os preceitos da Resolução 510/2016 (Brasil, 2016) que dispõe sobre as normas aplicáveis a atividades de pesquisa em Ciências Humanas e Sociais, assim como os princípios do Código de ética dos profissionais de Enfermagem, especialmente os artigos 56 e 58 do capítulo II dos deveres e artigos 97, 99, 100 e 101 do capítulo III das proibições (Cofen, 2017).

Apresenta-se o fluxo de seleção dos artigos, assim foram utilizadas as recomendações do PRISMA, sobre revisão sistemática, que propõe um fluxo de informação com as diferentes fases de uma revisão sistemática (Galvão et al., 2015), representado na Figura 1.

Figura 1 - Fluxograma baseado no PRISMA para estudos de revisão - Fluxo da informação com as diferentes fases de uma revisão sistemática, apresentando os artigos incluídos na revisão.

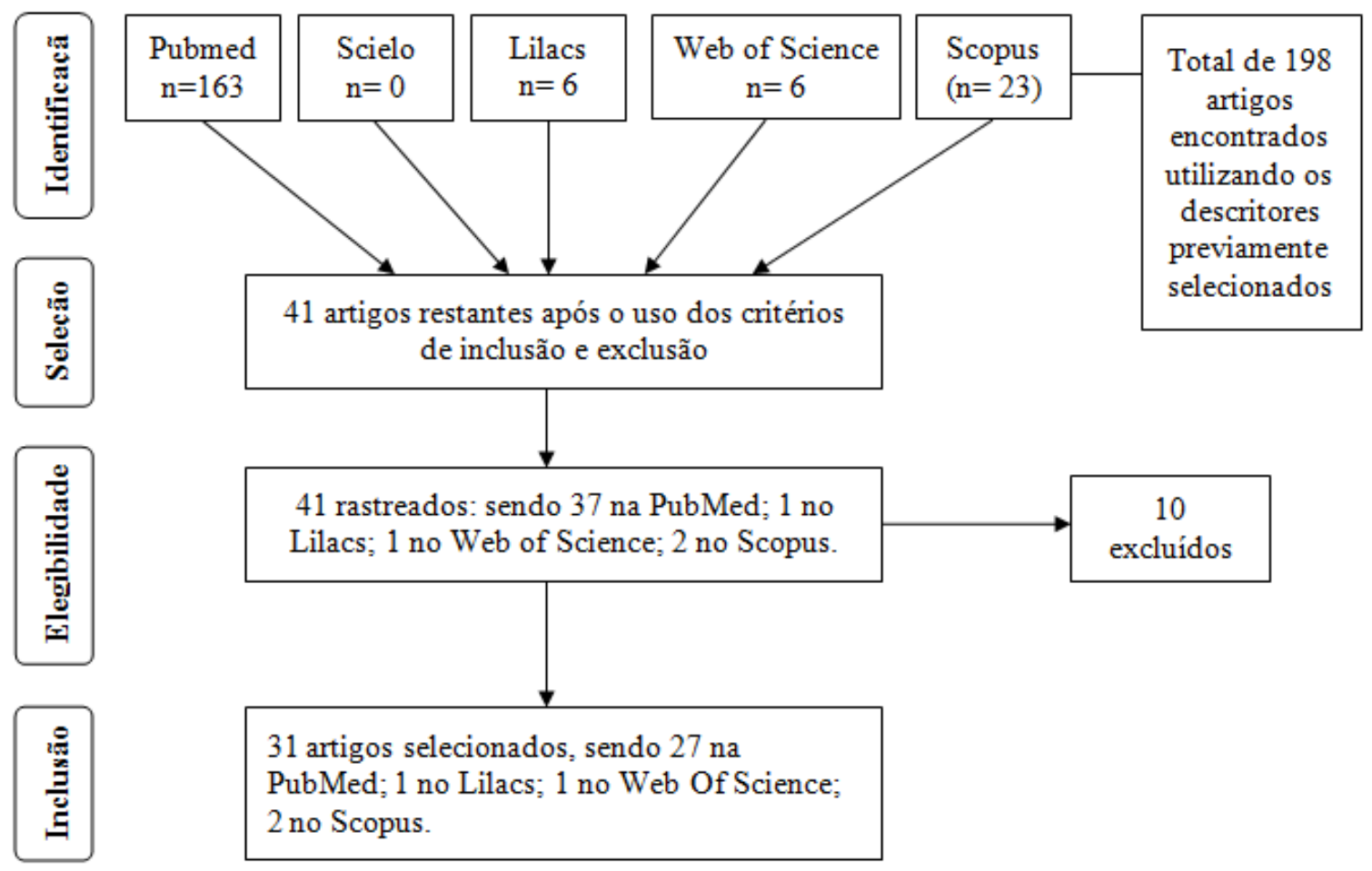

Fonte:Autores.

Dos quarenta e um artigos selecionados para leitura na íntegra, dez foram excluídos, com as seguintes justificativas: dois apenas caracterizavam os pacientes que não reinternaram; sete evidenciavam somente taxa de mortalidade, não englobando continuidade da assistência e um não tratava de continuidade ou cuidados após o IAM. 


\section{Resultados e Discussão}

O presente estudo de RI é composto por 31 artigos, os quais foram selecionados aplicando-se as etapas descritas no método. Quanto ao local de origem, verificou-se que 18 estudos foram publicados na América do Norte, nove artigos publicados na Europa; um na América Latina e três estudos na Ásia.

Em relação ao ano de publicação, 6,4 \% (2) foram publicados em 2015; 25,8\% (8) em 2016; 22,6\% (7) em 2017; 25,8\% (8) em 2018; 19,4\% (6) em 2019. Outrem, sobre os níveis de evidencia (NE), quatro artigos apresentaram NE II (Bonetti et al., 2019; Heaton et al., 2019; La Sala et al., 2015 \& Volpp et al., 2017,); dois artigos tiveram NE III (Munkhaugen et al., 2018 \& Hamar et al., 2016 ); 18 artigos demonstraram NE IV (Di Martino et al., 2016; Hickson et al., 2017; Rosenson et al., 2017; Rymer et al., 2018; Soto et al., 2018; Mahmoud et al., 2018; Schang et al., 2019; Kini et al., 2018; Chen et al., 2015; Chin et al., 2016; Wadhera et al., 2019; Marbach et al., 2018; Ventura et al., 2019; Fordyce et al., 2016; Sulo et al., 2017; Krumholz et al., 2016; Beckman et al., 2016 \& WASFY et al., 2018,), dois estudos evidenciaram NE V (Arakawa et al.,2016 \& Luu et al., 2019); e cinco artigos mostraram ter NE VI (Tung et al., 2017; Zabawa et al., 2018, Dharmarajan et al., 2017; Pocock et al., 2017 \& Pouche et al., 2016).

Respondendo ao objetivo da pesquisa identificaram-se três categorias quanto ao conteúdo, a primeira categoria intitulada: Identificação de fatores de risco associados às complicações após tratamento de IAM, foi composto por 11 artigos, representando 36\% do total de publicações; na segunda categoria: Estratégias para auxiliar na redução das taxas de readmissão hospitalares pós-tratamento de IAM foi composta por 10 artigos, o que corresponde a $32 \%$ e a terceira intitulada: Continuidade da assistência por meio de cuidados e gerenciamento da adesão medicamentosa, contou com 10 artigos, o que equivale a $32 \%$ do montante de publicações.

\section{Identificação de fatores de risco associados às complicações após tratamento de Infarto Agudo do Miocárdio}

Para que a continuidade do cuidado aos pacientes em fase de reabilitação se consolide, é necessário o levantamento de fatores de risco para eventuais complicações, visto que poderão embasar o planejamento de intervenções/ações adequadas.

Nesta perspectiva, Fordyce et al. (2016) destacam que pacientes com IAM, com parada cardíaca fora do hospital, apresentam maior risco de choque cardiogênico, insuficiência cardíaca, parada cardíaca recorrente, sangramento importante e necessidade de transfusão de hemácias em comparação com IAM sobreviventes sem parada cardíaca extra hospitalar, porém não interfere na mortalidade.

Identificou-se estudos que mencionam o Infarto, a Insuficiência cardíaca e a Pneumonia como condições sensíveis à readmissão hospitalar. Nesta perspectiva, Chin et al. (2016) e Dharmarajan et al. (2017) explicitam que estas condições aumentam o risco de readmissão em 30 dias. Neste sentido, o estudo de Wadhera et al. (2019) enfatizou que a redução na readmissão está atribuída a melhoria no planejamento da alta e nas transições de atendimento. Em consonância, Krumholz et al. (2016) destacaram que o tempo necessário para o risco diário de readmissão diminuir em $50 \%$ exige 20 dias após a hospitalização por IAM, o que sugere a importância de minimizar a vulnerabilidade a estas condições específicas, por meio do planejamento e acompanhamento pós alta.

Com isso, os dados reforçam a premissa de que acompanhar o paciente pós-tratamento de IAM possibilita aos profissionais de saúde compreender a capacidade do indivíduo de se adaptar à nova condição de saúde, seja com limitações funcionais, emocionais ou até mesmo a adesão a terapia medicamentosa.

Atrelado a este argumento, autores reforçam a necessidade da avaliação dos riscos (Mahmoud et al., 2018, Chen et al., 2015, Wasfy et al., 2018, Kini et al., 2018, Sulo et al., 2017), porém não se identificou semelhança entre os fatores associados as reinternações. Segundo Mahmoud et al. (2018) e Chen et al. (2015) os fatores que aumentam os riscos são o sexo feminino, diabetes mellitus, doença vascular periférica e insuficiência renal crônica. Em contraponto, Chen et al. (2015) destacam o 
diagnóstico prévio de IAM, aumento do tempo de internação hospitalar, desenvolvimento intra-hospitalar de fibrilação atrial, choque cardiogênico e insuficiência cardíaca como fatores de risco.

Para Wasfy et al. (2018) que desenvolveram modelos para estimar a probabilidade de um paciente com taxa de hospitalização prolongada e a necessidade de serviços pós-agudos, fatores como a idade avançada, insuficiência cardíaca na admissão, frequência cardíaca mais alta na admissão, choque na admissão, doença cerebrovascular e hemoglobina baixa, correspondem a riscos elevados de readmissão. Deste modo, é possível prever o tempo de permanência hospitalar do paciente e necessidade de cuidados pós-agudos, melhorando assim a qualidade do atendimento.

Assim como no estudo de Wasfy et al. (2018), Kini et al. (2018) mencionam a idade avançada como um agravante, assim como o diabetes mellitus e a insuficiência cardíaca, elencado como fator de risco no estudo de Chen et al. (2015). Kini et al. (2018) além de estruturarem os modelos de previsão para avaliar o risco de readmissão, identificaram como atenuante, a revascularização coronária.

Em consonância, Sulo et al. (2017) também trazem a insuficiência cardíaca como alto risco de mortalidade, o que representa um impacto negativo na sobrevida de pacientes após o IAM.Além disso, Beckman et al. (2016) evidenciaram que piores condições econômicas se configuram como um fator de risco, predominantemente para mulheres, o que pode repercutir na dificuldade em ter acesso a compra de medicamentos, com risco a agravar sua condição de saúde pós IAM.

Em outro estudo, Pocock et al. (2017) elaboraram um sistema de pontuação de risco para a sobrevida individual do paciente em dois anos, onde foram testados e aplicados 17 preditores independentes, como a idade, revascularização coronariana/trombólise, creatinina sérica elevada, hemoglobina baixa, doença cardíaca prévia obstrutiva ou pulmonar obstrutiva crônica, glicemia elevada, uso de diuréticos ou inibidor de aldosterona na alta, sexo masculino, baixa escolaridade, complicações cardíacas hospitalares, baixo índice de massa corporal, diagnóstico de infarto do miocárdio com supradesnivelamento do segmento ST e classe de Killip. Este sistema facilita a identificação de pacientes de alto risco, ajudando assim a orientar medidas adequadas para prevenção secundária.

A aplicação dos modelos mostrou-se altamente efetiva, pois permite a identificação dos pacientes com prognóstico desfavorável, o que facilita o direcionamento de intervenções para pacientes com maior risco de readmissão, sobretudo em levantamento de medidas prioritárias de prevenção secundária para aumentar a sobrevida. A pouca similaridade entre os fatores amplia um leque de possíveis fatores de risco às complicações desfavoráveis, porém, desperta incertezas quanto ao direcionamento de melhores práticas de atenção às pessoas após o tratamento do IAM

\section{Estratégias para auxiliar na redução das taxas de readmissão hospitalares pós-tratamento de IAM}

Com o intuito de reduzir as taxas de readmissão após o tratamento da fase aguda, é imprescindível que sejam adotadas estratégias direcionadas ao cuidado longitudinal, com base nas principais complicações existentes após o tratamento do IAM.

Como estratégia para reduzir as taxas de readmissão, Rymer et al. (2018), Tung et al. (2017) e Zabawa et al. (2018) avaliaram a relevância do acompanhamento médico para qualificar a continuidade do cuidado após o tratamento hospitalar do IAM, no entanto, apenas um estudo realizado na China (Tung et al., 2017) identificou esta relação com a diminuição da readmissão em 30 dias.

Outro método evidenciado foi a implementação de programas para reduzir a readmissão hospitalar, voltados principalmente para a transição dos cuidados após a fase aguda do IAM (Soto et al., 2018, Arakawa et al., 2016, Hamar et al.,2016, Marbachet al., 2018, Wadhera et al., 2019). No Japão, um estudo desenvolvido por Arakawa et al. (2016) fez uma investigação nacional a respeito da aplicabilidade de um programa que visa estabelecer uma conexão entre a fase aguda e o gerenciamento da doença na fase crônica para o IAM, porém, a oferta de reabilitação cardíaca ambulatorial foi de apenas 18\%, o que poderia comprometer a melhoria do prognóstico a longo prazo. 
Manejos de prevenção de complicações associadas a adesão medicamentosa foram desenvolvidos por farmacêuticos (Heaton et al., 2019\&Bonetti et al., 2019) que estruturaram programas de aconselhamento após a alta hospitalar. Nesta conjuntura, Heaton et al. (2019) em um estudo randomizado objetivaram avaliar o impacto de um programa de gerenciamento de terapia medicamentosa fornecido por farmacêuticos nas taxas de readmissão de 30 dias 45 após a alta. O programa consiste em realizar uma consulta presencial com o paciente, onde será desenvolvida uma educação em saúde, esclarecendo sobre os efeitos da medicação e sobre quais os sinais de alerta que indicam que o estado de saúde está se deteriorando. Uma semana depois os farmacêuticos fizeram o acompanhamento novamente pessoalmente ou por telefone e após todas as recomendações de medicamentos eram comunicadas ao médico de cuidados primários via fax.

Os pacientes no grupo de intervenção foram significativamente menos propensos a experimentar uma readmissão com a utilização deste programa. Deste modo, a transmissão das informações do paciente do ambiente de internação para a farmácia comunitária é essencial para a transição dos pacientes com sucesso.

Em conformidade, estudo realizado no Brasil (Bonetti et al., 2019) analisou um programa de aconselhamento de alta, conduzido por farmacêutico, que consistia na realização de consultas com os pacientes ou seus cuidadores 30 dias após a alta, também voltado para a educação em saúde, incluindo questões sobre doses, esquema posológico, duração da terapia e possíveis eventos adversos. Posteriormente, os pacientes eram contatados por telefone pelo mesmo farmacêutico 3 e 15 dias após a alta, para reforço da sessão de aconselhamento anterior. O programa demonstrou muitos benefícios, pois os pacientes apresentaram menos problemas de farmacoterapia nos 30 dias pós-alta, principalmente relacionados à administração de medicamentos e adesão.

Para diminuir o risco de readmissão e mortalidade Munkhaugen et al. (2018), analisaram a prevalência do controle dos fatores de risco incluindo como as barreiras clínicas relacionadas ao paciente e ao tratamento o tabagismo, colesterol de lipoproteína de baixa densidade, pressão arterial, obesidade, exercícios físicos e diabetes. O estudo concluiu que é possível melhorar o perfil de risco de muitos pacientes com a introdução de medidas relativamente simples, como medição da 46 pressão arterial e terapia de reposição de nicotina auxiliando no processo de parar de fumar, além do encaminhamento para programas de reabilitação cardíaca (Munkhaugen et al., 2018).

Em consonância, na França foi efetivado um estudo sobre o efeito da prescrição de Reabilitação Cardíaca (RC) na mortalidade em cinco anos em pacientes com infarto agudo do miocárdio após a alta, evidenciando que a prescrição de RC diminuiu substancialmente a mortalidade. Portanto, o estudo concluiu que, principalmente medicamentos e RC, além de reduzirem a mortalidade, melhoram a qualidade de vida e reduzem o número de reinternações hospitalares (Pouche et al., 2016).

Estima-se que se o paciente infartado que recebe um tratamento adequado e precoce poderá ser beneficiado com menores riscos de complicações, como a insuficiência cardíaca. Em consonância, no que tange ao acompanhamento após o tratamento acredita-se que o rigor desta atenção e o direcionamento às reais necessidades do indivíduo, também possa ser um diferencial, com significativa melhoria do prognóstico.

\section{Continuidade da assistência por meio de cuidados e gerenciamento da adesão medicamentosa}

Para que se obtenha melhores resultados de saúde o acompanhamento com profissionais qualificados e preocupados com a aceitação do paciente frente à sua condição de saúde é essencial, promovendo também a melhora do prognóstico e expectativa de vida. Nesta perspectiva, a adesão medicamentosa se constitui como uma estratégia fundamental para que se atinja melhores resultados de saúde.

O estudo de Ventura et al. (2019) elucida que a sobrevida em um ano após o infarto está associada a adesão ao tratamento farmacológico na fase pós-aguda, em todos os gêneros e em idoso. Estes resultados reforçam a necessidade de 
melhorar o desempenho nas diferentes fases do cuidado, garantindo melhoraria na expectativa de vida.

A adesão medicamentosa foi destacada como estratégia direta para reduzir as taxas de readmissão (Heaton et al., 2019\&Bonetti et al., 2019), assim como uma maneira de oferecer cuidado na continuidade da assistência (Rosenson et al., 2017; Luu et al., 2019; Volpp et al., 2017; DiMartino et al., 2016\&Hickson et al.,2017), o que contribui para estabilização clínica e sobrevida.

Para comparar o benefício oferecido por uma prevenção secundária intensiva estruturada, La Sala et al. (2015) realizaram um estudo no qual os enfermeiros usavam um formulário inovador de enfermagem multidimensional, levando em consideração tanto fatores biológicos, quanto fatores psico-sócio-relacionais. Esta intervenção mostrou ser eficaz em diversos aspectos como: identificar doenças e fatores que prevêem algum problema, permitindo assim a personalização de intervenções;orientar a avaliação dos fatores de risco e adesão ao tratamento; manter a continuidade do atendimento entre o hospitale em casa com o objetivo de melhorar os resultados de saúde aumentando a adesão ao tratamento e reduzindo a incidência de novo infarto e reinternações.

Ainda em relação à adesão, Rosenson et al. (2017) objetivaram examinar as tendências e os preditores do uso de estatina de alta intensidade após a alta 47 hospitalar por infarto do miocárdio. Segundo o estudo, embora tenha ocorrido um aumento nas prescrições de estatinas alta densidade, ainda houve uma porcentagem substancial de estatinas de baixa e moderada densidade. Os autores destacam a necessidade de continuar o estímulo do uso de estatina de alta intensidade após a alta hospitalar por IAM, visto que esta é considerada mais eficaz para prevenir eventos recorrentes de doença cardiovascular (Rosenson et al., 2017).

Luu et al. (2019) em uma pesquisa transversal, concluíram que principais fatores que afetam a adesão à terapia antiplaquetária entre pacientes com diagnóstico de IAM são a distância de casa ao hospital, conhecimento do paciente sobre o tratamento correto e baixa renda. Logo, o estudo concluiu que aumentar a interação entre médicos e pacientes é essencial para melhorar o conhecimento dos pacientes, contribuindo assim para um tratamento eficaz.

Com o objetivo de melhorar a adesão ao tratamento farmacológico, Volpp et al. (2017) realizaram uma intervenção que consistia em disponibilizar frascos eletrônicos com monitoramento da abertura, para pacientes com IAM que tiveram alta. No entanto, a média de adesão à medicação não diferiu entre o grupo controle e a intervenção.

Di Martino et al. (2016) relataram em seu estudo que, na prática clínica, a farmacoterapia de prevenção secundária após IAM não é consistente com as diretrizes clínicas. Seus resultados demonstraram que a adesão foi influenciada mais pelo hospital que deu alta ao paciente do que pelos prestadores de cuidados primários, no entanto sugerem que é necessário estimular a associação de médicos da atenção primária, a fim de melhorar a continuidade do atendimento.

Em consonância com este achado, Hickson et al. (2017) avaliaram se o acompanhamento pós alta com um provedor de cuidados primários (PCP) e/ou cardiologista está associado a mudança na adesão as estatinas após um IAM. Foi evidenciado que os pacientes que seguiram apenas com um PCP tiveram probabilidade de um aumento de adesão. Acompanhamento com um cardiologista ou ambos os tipos de provedores tiveram uma associação mais forte com um aumento na adesão. Deste modo infere-se para melhorar a adesão a terapia medicamentosa após um IAM, é essencial que seja realizado um cuidado coordenado com médicos ambulatoriais, melhorando assim a saúde do paciente.

Schang et al. (2019) também investigaram o papel dos hospitais e médicos em consultório em redes que prestam cuidados à mesma população no que diz respeito à prestação oportuna de cuidados adequados após a alta hospitalar e concluíram que a prescrição apropriada de farmacoterapia de prevenção secundária após IAM está sujeita a variação de prática entre hospitais e médicos. Em vista disso, é imprescindível fortalecer a capacidade do hospital para gerenciamento de alta e coordenação entre hospitais e médicos em consultório, garantindo assim a continuidade do cuidado aos pacientes.

Pode-se supor que o acompanhamento com profissionais qualificados e preocupados com a aceitação do paciente 
frente sua condição de saúde permitirá melhores resultados de saúde, promovendo também a melhora do prognóstico e expectativa de vida.

Ao realizar a análise crítica dos estudos que mencionavam sobre a importância do cuidado após a fase aguda do IAM, observou-se que a enfermagem foi pouco mencionada neste processo. Neste contexto de cuidado longitudinal, o Brasil conta com a Política Nacional de Atenção Básica (Brasil, 2017), que traz a continuidade da assistência como princípio básico a ser operacionalizado no atendimento ao paciente.

A continuidade de cuidados é um direito dos cidadãos que assume ainda maior importância quando estes requerem intervenções multiprofissionais dirigidos para as situações crônicas ou complexas de que são portadores. Esta continuidade deve iniciar-se no serviço onde a pessoa é atendida, o que exige a necessidade de se efetivarem contatos e de operacionalizar um plano de alta adequado (Mendes et al., 2017).

Neste cenário, há uma necessidade de um cuidado humanizado, acolhedor e direcionado às necessidades dos sujeitos. Segundo Kahl et al. (2018) o modelo centrado no cuidado médico vem sendo substituído, e a enfermagem se destaca como fundamental para a integralidade do cuidado, a longitudinalidade e a melhoria da qualidade da atenção à saúde dos indivíduos.

Deste modo, destaca-se a necessidade da participação dos agentes de saúde e da equipe de enfermagem, sobretudo do enfermeiro, pois seu envolvimento é essencial para a efetivação de estratégias de atenção à saúde, como a organização das atividades, o funcionamento do centro de saúde e assistência direta à saúde (Kahl et al., 2018).Além disso, este profissional é responsável por realizar a ligação entre a família, os outros profissionais e os recursos da comunidade, promovendo uma maior equidade no acesso aos cuidados de saúde, uma maior proximidade com o cidadão e a continuidade de cuidados (Mendes et al., 2017).

Nesta categoria, a adesão ao tratamento farmacológico se destaca como estratégia para aumento da sobrevida. Deste modo, é imprescindível que a equipe multidisciplinar forneça as informações adequadas para a implementação e manutenção da terapia medicamentosa, diminuindo assim o risco de complicações.

\section{Considerações Finais}

Ao analisar as produções que compuseram a amostra deste estudo evidenciou-se um amplo engajamento dos autores na elaboração de estratégias para reduzir a readmissão hospitalar, assim como delinear planos de cuidados. Verificou-se que a aplicação de modelos de predição de fatores de risco apresentou resultados satisfatórios, pois permitiu a identificação de pacientes com prognóstico desfavorável, auxiliando no planejamento de intervenções e de medidas adequadas de prevenção, o que favorece sua sobrevida.

Em relação à diminuição das taxas de readmissão hospitalar, identificou-se como principal estratégia a criação e implementação de programas voltados à transição dos cuidados após a fase aguda do IAM contribuindo para a efetivação da continuidade dos cuidados. Neste contexto, também se observou que para estabelecer a continuidade da assistência, as consultas periódicas precisam ser mantidas, assim como o adequado aconselhamento e gerenciamento da terapia medicamentosa, visto que os estudos demonstraram que grande parte dos problemas dos pacientes esteve voltada para a adesão aos medicamentos.

Ressalta-se que apenas um estudo trouxe destaque à enfermagem como protagonista deste processo de cuidado longitudinal. No entanto, destaca-se aqui a importância da enfermagem no processo de transição de cuidados, visto que o enfermeiro é o profissional que está à frente do cuidado e deste modo, torna-se fundamental a elaboração da assistência por meio do planejamento de intervenções, orientações e práticas de prevenção.

Identifica-se como potencialidade deste estudo a exposição de estratégias de redução da readmissão hospitalar assim como de continuidade do cuidado passíveis de serem implementadas no Brasil, na medida em que se tratam de práticas 
acessíveis e de baixo custo. Além disso, a elaboração deste estudo poderá contribuir para dar maior visibilidade ao paciente pós-tratamento de IAM, os pacientes carecem de um acompanhamento longitudinal para prevenir complicações e oferecer o manejo necessário.

Como limitação verificou-se a impossibilidade de conhecer o método de cuidado no Brasil, pois foi encontrado somente um estudo voltado a temática do estudo no país. Pode-se inferir que é uma temática incipiente no campo da pesquisa, porém acredita-se que seja uma prática assistencial também do enfermeiro, inserido na equipe de saúde. Assim, a temática carece de ampla discussão, visto que a continuidade do cuidado pós IAM mostrou-se efetiva de acordo com as publicações internacionais.

\section{Agradecimentos}

À Coordenação de Aperfeiçoamento de Pessoal de Nível Superior (CAPES) pela bolsa de doutorado da segunda e quarta autora.

\section{Referências}

Arakawa, T., Kumasaka, L., Nakanishi, M., Nagayama, M., Adachi, H., Ikeda, K., Fujimoto, K., Tashiro, T., Momomura, S., \& Goto, Y. (2016). Regional Clinical Alliance Path and Cardiac Rehabilitation After Hospital Discharge for Acute Myocardial Infarction Patients in Japan - A Nationwide Survey -. Circulation Journal, 80(8), 1750-1755. https://doi.org/10.1253/circj.CJ-15-1392

Baratier, T., \& Marcon, S. S. (2011). Longitudinalidade do cuidado: compreensão dos enfermeiros que atuam na estratégia saúde da família. Esc Anna Nery, 15(04), 802-10. http://dx.doi.org/10.1590/S1414-81452011000400020

Bardin, L. (2013). Análise de conteúdo. Edições 70.

Beckman, A. L., Bucholz, E., Zhang, w., Xu, X., Dreyer, R. P., Strait, K. M., Spertus, J. A., Krumholz, H., \& Spatz, E. S. (2016). Sex differences in financial barriers and the relationship to recovery after acute myocardial infarction. Journal of the American Heart Association, 5(10), 1-15. https://doi.org/10.1161 / JAHA.116.003923

Bonetti, A. F., Bagatim, B. Q., Bottacin, W. E., Mendes, A. M., Rotta, I., Reis, R. C., Fávero, M. L. D., Fernandez-Llimos, F \&Pontarolo, R. (2019). Pharmacotherapy problems in cardiology patients 30 days post discharge from a tertiary hospital in Brazil: A randomized controlled trial. Clinics 74, e1091. https://doi.org/10.6061/clinics/2019/e1091

Chen, H.Y., Tisminetzky, M., Yarzebski, J., Gore, J. M., \& Goldberg, R. J. (2016). Decade-long Trends in the Frequency of 90-day Rehospitalizations after Hospital Discharge for Acute Myocardial Infarction. The American journal of cardiology, 117(5), 743-748. https://doi.org/10.1016/j.amjcard.2015.12.006

Chin, D. L., Bang, H., Manickam, R. N., \& Romano, P. S. (2016). Rethinking Thirty-Day Hospital Readmissions: Shorter Intervals Might Be Better Indicators Of Quality Of Care. Health Affairs, 35(10), 1867-1875.Doi: https://doi.org/10.1377/hlthaff.2016.0205

Cofen. Conselho Federal de Enfermagem. (2017). Resolução COFEN no 564/2017. Aprova o novo Código de Ética dos Profissionais de Enfermagem.

Di Martino, M. D., Alagna, M., Cappai, G., Mataloni, F., Lallo, A., Perucci, C. A., Davoli, M \& Fusco, D. (2016). Adherence to evidence-based drug therapies after myocardial infarction: Is geographic variation related to hospital of discharge or primary care providers? A cross-classified multilevel design. BMJ Open, 6(4), e010926. https://doi.org/10.1136/bmjopen-2015-010926

Dharmarajan, K., Wang, Y., Lin, Z., Normand, S.-L. T., Ross, J. S., Horwitz, L. I., Desai, N. R., Suter, L. G., Drye, E. E., Bernheim, S. M., \& Krumholz, H. M. (2017). Association of Changing Hospital Readmission Rates With Mortality Rates After Hospital Discharge. JAMA, 318(3), 270-278. https://doi.org/10.1001/jama.2017.8444

Hamar, B., Rula, E. Y., Wells, A. R., Coberley, C., Pope, J., \&Varga, D. (2016). Impact of a scalable care transitions program for readmission avoidance. The American journal of managed care, 22(1), 28-34.

Heaton, P. C., Frede, S., Kordahi, A., Lowery, L., Moorhead, B., Kirby, J., Kunze, N., \&Luder, H. (2019). Improving care transitions through medication therapy management: A community partner ship to reduce readmissions in multiple health-systems. Journal of the American Pharmacists Association, 59(3), 319-328. https://doi.org/10.1016/j.japh.2019.01.005

Hickson, R. P., Robinson, J. G., Annis, I. E., Killeya-Jones, L. A., Korhonen, M. J., Cole, A. L., \&Fang, G. (2017). Changes in Statin Adherence Followingan Acute Myocardial Infarction Among Older Adults: Patient Predictors and the Association With Follow-Up With Primary Care Providers and/or Cardiologists. Journal of the American Heart Association, 6(10). https://doi.org/10.1161/JAHA.117.007106

Fordyce, C. B., Wang, T. Y., Chen, A. Y., Thomas, L., Granger, C. B., Scirica, B. M., Henry, T. D., Wong, G. C., Ramanathan, K., Hansen, C. M., Kragholm, K., Peterson, E. D \& Anderson, M. L. (2016). Long-Term Post-Discharge Risks in Older Survivors of Myocardial Infarction With and Without Out-ofHospital Cardiac Arrest. Journal of the American College of Cardiology, 67(17), 1981-1990. https://doi.org/10.1016/j.jacc.2016.02.044

Galvão, T. F., Pansani, T. S. A., \&Harrad, D. (2015). Principais itens para relatar Revisões sistemáticas e Meta-análises: A recomendação PRISMA. Epidemiologia e Serviços de Saúde, 24(2), 335-342. http://dx.doi.org/10.5123/S1679-49742015000200017 
Kahl, C., Meirelles, B. H. S., Lanzoni, G. M. M., Koerich, C., \& Cunha, K. S. (2018). Ações e interações na prática clínica do enfermeiro na Atenção Primária à Saúde. RevEscEnfermUSP, 52, :e03327. http://dx.doi.org/10.1590/S1980-220X2017025503327

Kessler, M., Lima, S. B. S. de, Weiller, T. H., Lopes, L. F. D., Ferraz, L., Eberhardt, T. D., Soares, R. S. de A \& Trindade, L. de L. (2019). Longitudinalidade do cuidado na atenção primária: Avaliação na perspectiva dos usuários. Acta Paul Enferm., 32(2), 186-193. https://doi.org/10.1590/1982-0194201900026

Kini, V., Peterson, P. N., Spertus, J.A., Kennedy, K.F., Arnold, S.V., Wasfy, J. H., Curtis, J. P., Bradley, S.M., Amin, A.P., Michael H \&Masoudi, F.A. (2018). Clinical Model to Predict 90-Day Risk of Readmission After Acute Myocardial Infarction. Circulation. Cardiovascular quality and outcomes,11(10), e004788. https://doi.org/10.1161/ CIRCOUTCOMES.118.004788

Krumholz, H. M., Hsieh A., Dreyer, R. P, Welsh J, Desai, N. R, Dharmarajan K. (2016). Trajectories of risk for specific readmission diagnoses after hospitalization for heart failure, acute myocardial infarction, or pneumonia. PLoS ONE, 11(10), 1-14. https://doi.org/10.1371/journal.pone.0160492.

La Sala, R., Foà, C., Paoli, G., Mattioli, M., Solinas, E., Artioli, G., \&Ardissino, D. (2015). Multi-dimensional nursing form: A novel means of approaching nurse-led secondary cardiology prevention. Acta Bio-Medica: AteneiParmensis, 86(3), 174-182.

Luu, N. M., Dinh, A. T., Nguyen, T. T. H., \& Nguyen, V. H. (2019). Adherence to Antiplatelet Therapy after Coronary Intervention among Patients with Myocardial Infarction Attending Vietnam National Heart Institute. BioMedResearch International, 2019, 6585040. https://doi.org/10.1155/2019/6585040

Mahmoud, A. N., Elgendy, I. Y., Mojadidi, M. K., Wayangankar, S. A., Bavry, A. A., Anderson, R. D., Jneid, H \&Pepine, C. J. (2018). Prevalence, Causes, and Predictors of 30-Day Readmissions Following Hospitalization With Acute Myocardial Infarction Complicated By Cardiogenic Shock: Findings From the 2013-2014 National Readmissions Database. Journal of the American Heart Association, 7(6). https://doi.org/10.1161/JAHA.117.008235

Marbach, J. A., Johnson, D., Kloo, J., Vira, A., Keith, S., Kraft, W. K., Margules, N., \&Whellan, D. (2018). O impacto de um programa de transição de cuidados nas taxas de readmissão de infarto agudo do miocárdio. American Journalof Medical Quality, 33(5), 481-486. https://doi.org/10.1177/1062860618754702

Mendes, Felismina Rosa P., Gemito, Maria Laurência G. Parreirinha, Caldeira, Ermelinda do Carmo, Serra, Isaura da Conceição, \& Casas-Novas, Maria Vitória. (2017). A continuidade de cuidados de saúde na perspetiva dos utentes. Ciência \& Saúde Coletiva, 22(3), 841-853. https://doi.org/10.1590/141381232017223.26292015

Mendes, K. D. S., Silveira, R. C. de C. P \& Galvão, C. M. (2019). Use ofthebibliographicreference manager in theselectionofprimarystudies in integrative reviews. Texto\&amp; Contexto - Enfermagem, 28, e20170204Doi: https://doi.org/10.1590/1980-265x-tce-2017-0204

Ministério da saúde. (2011). Atenção Primária e Promoção da Saúde. Conselho Nacional de Secretários de Saúde. CONASS, 197.

Ministério da saúde. (2016). Resolução n $\mathrm{n}^{\circ}$ 510, de 7 de abril de 2016. Dispõe sobre as normas aplicáveis a pesquisas em Ciências Humanas e Sociais. Conselho Nacional de Saúde. Diário Oficial da União, Brasília.

Munkhaugen, J., Peersen, K., Sverre, E., Gjertsen, E., Gullestad, L., Dammen, T., Husebye, E., \&Otterstad, J. E. (2018). The follow-up after myocardial infarction - is it good enough? Tidsskrift for Den norske legeforening. https://doi.org/10.4045/tidsskr.17.1050

Pocock, S. J., Huo, Y., Van de Werf, F., Newsome, S., Chin, C. T., Vega, A. M., Medina, J., \& Bueno, H. (2017). Predicting two-year mortality from discharge after acute coronary syndrome: Aninternationally-basedrisk score. European Heart Journal. Acute Cardiovascular Care, 8(8), $727-737$. https://doi.org/10.1177/2048872617719638

Pouche, M., Ruidavets, J. B., Ferrières, J., Iliou, M. C., Douard, H., Lorgis, L., Carrié, D., Brunel, P., Simon, T., Bataille, V., \&Danchin, N. (2016). Cardiac rehabilitation and 5-year mortality after acute coronary syndromes: The 2005 French FAST-MI study. Archives of Cardiovascular Diseases, 109 (3), $178-187$. https://doi.org/10.1016/j.acvd.2015.09.009

Rosenson, R. S., Farkouh, M. E., Mefford, M., Bittner, V., Brown, T. M., Taylor, B., Monda, K. L., Zhao, M., Dai, M., \&Muntner, P. (2017). Trends in Use of High-Intensity Statin Therapy After Myocardial Infarction, 2011 to 2014. Journal of the American College of Cardiology, 69(22), 2696-2706. https://doi.org/10.1016/j.jacc.2017.03.585

Rymer, J. A., Chen, A. Y., Thomas, L., Stafford, J., Enriquez, J. R., Goyal, A., Peterson, D. E., \&Wang, T. Y. (2018). Advanced practice provider versus physician-only outpatient follow-up after acute myocardial infarction. Journal of the American Heart Association, 7(17), 1-9. http://doi.org/10.1161/JAHA.117.008481

Schang, L., Koller, D., Franke, S., \&Sundmacher, L. (2019). Exploring the role of hospitals and office-based physicians in timely provision of statins following acute myocardial infarction: A secondary analysis of a nation wide cohort using cross-classified multilevel models. BMJ Open, 9(10). https://doi.org/10.1136/bmjopen-2019-030272

Schimith, M. D., Brêtas, A. C. P., Budó, M. de L. D., Simon, B. S., Leal, T. C \&Backes, D. M. (2014). Continuidade do cuidado na Rede de Atenção à Saúde: Negociação entre usuários e profissionais. Rev Rene, 15(5), Article 5. http://www.periodicos.ufc.br/rene/article/view/3251

Simão, A. F., Precoma, D. B., Andrade, J. P., Correa Filho, H., Saraiva, J. F. K., Oliveira, G. M. M., Murro, A. L. B., Campos, A., Alessi, A., Avezum Junior, A., Achutti, A. C., Miguel, A., Sousa, A. C. S., Lotemberg, A. M. P., Lins, A. P., Falud, A. A., Brandão, A. A., Sanjuliani, A. F., Sbissa, A. S\& Souza, W. (2013). I Diretriz Brasileira de Prevenção Cardiovascular. Arquivos Brasileiros de Cardiologia, 101(6), 1-63. https://doi.org/10.5935/abc.2013S012

Siqueira, D. A., Costa Junior, J. R \& Abizaid, A. (2016). Estratégia invasiva nas síndromes coronarianas agudas sem supradesnivelamento do segmento st. Rev. Soc. Cardiol. 99-104.

Soto, G. E., Huenefeldt, E. A., Hengst, M. N., Reimer, A. J., Samuel, S. K., Samuel, S. K \&Utts, S. J. (2018). Implementation and impact analysis of a transitional care pathway for patients presenting to the emergency department with cardiac-related complaints. BMC Health Services Research, 18(1), 672. https://doi.org/10.1186/s12913-018-3482-2 
Sulo, G., Igland, J., Nygård, O., Vollset, S. E., Ebbing, M., Poulter, N., Egeland, G. M., Cerqueira, C., Jørgensen, T \& Tell, G. S. (2017). Prognostic Impact of In-Hospital and Post discharge Heart Failure in Patients With Acute Myocardial Infarction: A Nationwide Analysis Using Data From the Cardiovascular Disease in Norway (CVDNOR) Project. Journal ofthe American Heart Association, 6(3). https://doi.org/10.1161/JAHA.116.005277

Stillwell, B. S. B., Fineout-Overholt, E., Melnyk, B. M., \& Williamson, K. M. (2010).Searching for the Evidence: Strategies to help you conduct a successful search. AJN, $110(5), 41-47$.

Troncoso, L. T., Oliveira, N. C. C., Leporaes, R. A., Eira, T. L \& Pinheiro, V. P. (2018). Estudo epidemiológico da incidência do infarto agudo do miocárdio na população brasileira. Cadernos da Medicina - UNIFESO, 1(1), 91-101.

Tung, Y. C., Chang, G. M., Chang, H. Y \& Yu, T. H. (2017). Relation ship between Early Physician Follow-Up and 30-Day Readmission after Acute Myocardial Infarction and Heart Failure. PloSOne, 12(1), e0170061. https://doi.org/10.1371/journal.pone.0170061

Ursi, E. S. (2005). Prevenção de lesões de pele no perioperatório: Revisão integrativa da literatura. Dissertação (Mestrado em Enfermagem) - Escola de Enfermagem, Universidade de São Paulo, Ribeirão Preto, 130.

Vargas, R. A. de, Riegel, F., Oliveira Junior, N. de, Siqueira, D. S \&Crossetti, M. da G. O. (2017). Qualidade de vida de pacientes pós-infarto do miocárdio: Revisão integrativa da literatura. Rev. enferm. UFPE online, 11(7), 2803-2809. 10.5205/reuol.10939-97553-1-RV.1107201721

Ventura, M., Belleudi, V., Sciattella, P., Domenicantonio, R. D., Martino, M. D., Agabiti, N., Davoli, M \& Fusco, D. (2019). High qualityprocessofcareincreasesone-yearsurvivalafteracutemyocardialinfarction (AMI): A cohort study in Italy. Plos One, 14(2), e0212398. https://doi.org/10.1371/journal.pone.0212398

Volpp, K. G., Troxel, A. B., Mehta, S. J., Norton, L., Zhu, J., Lim, R., Wang, W., Marcus, N., Terwiesch, C., Caldarella, K., Levin, T., Relish, M., Negin, N., Smith-McLallen, A., Snyder, R., Spettell, C. M., Drachman, B., Kolansky, D \&Asch, D. A. (2017). Effectof Electronic Reminders, Financial Incentives, and Social Supporton Outcomes After Myocardial Infarction: The Heart Strong Randomized Clinical Trial. JAMA Internal Medicine, 177(8), 1093-1101. https://doi.org/10.1001/jamainternmed.2017.2449

Wadhera, R. K., Maddox, K. E., Kasi, D. S., Shen, C., \&Yeh, R. (2019). Hospital revisits within 30 days after discharge for medical conditions targeted by the Hospital Readmissions Reduction Program in the United States: National retrospective analysis. The BMJ, 366, 1-6. https://doi.org/10.1136/bmj.14563

Wasfy, J. H., Kennedy K. F., Masoudi, F. A., Ferris, T. G., Arnold, S. V., Kini, V., Peterson, P., Curtis, J. P., Amin, A. P., Bradley, S. M., French, W. J., Messenger, J., Ho, P.M\&Spertus, J.A. (2018). Predicting Length of Stay and the Need for Postacute Care After Acute Myocardial Infarction to Improve Healthcare Efficiency. Circulation. Cardiovascular quality and outcomes, 11(9), 1-13. https://doi.org/10.1161/CIRCOUTCOMES.118.004635

Zabawa, C., Cottenet, J., Zeller, M., Mercier, G., Rodwin, V. G., Cottin, Y \&Quantin, C. (2018). Thirty-day rehospitalizations among elderly patients with acute myocardial infarction: Impact of postdischarge ambulatory care. Medicine, 97(24), e11085. https://doi.org/10.1097/MD.0000000000011085 